\title{
Clinically Meaningful Blood Pressure Reductions With Low Intensity Isometric Handgrip Exercise. A Randomized Trial
}

\author{
N. C. L. HESS ${ }^{1}$, D. J. CARLSON ${ }^{1}$, J. D. INDER ${ }^{1}$, E. JESULOLA ${ }^{1}$, J. R. MCFARLANE ${ }^{1}$, \\ N. A. SMART ${ }^{1}$
}

${ }^{1}$ School of Science and Technology, University of New England, Armidale, Australia

Received July 2, 2015

Accepted January 8, 2016

On-line April 12, 2016

\begin{abstract}
Summary
There exists no examination of what is the minimum antihypertensive threshold intensity for isometric exercise training. Twenty two normotensive participants were randomly assigned to training intensities at either $5 \%$ or $10 \%$ of their maximal contraction. Twenty participants completed the study. Clinical meaningful, but not statistically significant, reductions in systolic blood pressure were observed in both $5 \%$ and $10 \%$ groups $-4.04 \mathrm{~mm} \mathrm{Hg} \quad(95 \% \mathrm{CI} \quad-8.67$ to $+0.59, p=0.08)$ and $-5.62 \mathrm{~mm} \mathrm{Hg}(95 \% \mathrm{CI}-11.5$ to $+0.29, p=0.06)$ respectively after 6 weeks training. No diastolic blood pressure reductions were observed in either $5 \%-0.97 \mathrm{~mm} \mathrm{Hg}(95 \% \mathrm{CI}-2.56$ to +0.62 , $p=0.20$ ) or $10 \% \mathrm{MVC}+1.8 \mathrm{~mm} \mathrm{Hg}$ (95\% CI -1.29 to +4.89 , $p=0.22$ ) groups respectively after training. In those unable to complete isometric exercise at the traditional $30 \%$ intensity, our results suggest there is no difference between 5 and $10 \%$ groups and based on the principle of regression to the mean, this could mean both interventions induce a similar placebo-effect.
\end{abstract}

\section{Key words}

Blood pressure • Isometric exercise • Hypertension

\section{Corresponding author}

N. Smart, School of Science and Technology, University of New England, Armidale, NSW 2350, Australia. E-mail: nsmart2@une.edu.au

\section{Introduction}

Hypertension is considered to be the greatest modifiable risk factor pertaining to CVD (Hajjar and Kotchen 2003). Health professionals recommend lifestyle modifications such as exercise for the prevention and treatment of hypertension in both normotensive and hypertensive individuals (James et al. 2014), traditionally, aerobic exercise training that targets cardiovascular fitness has been the first line exercise prescription for managing hypertension.

Isometric exercise training (IET) involves a single sustained muscle contraction against an immovable load or resistance with no, or minimal, change in length of the involved muscle group. Whilst the physiological mechanisms elicited by IET are not entirely clear there is a growing body of evidence that supports the role of IET to accommodate significant reductions in resting systolic and diastolic blood pressures in both hypertensive and normotensive men and women (Millar et al. 2008, Wiles et al. 2010, Devereux et al. 2011, Badrov et al. 2013a,b). Results of a recent meta-analyses reported that compared to dynamic aerobic endurance training, dynamic resistance training, and training comprising both dynamic endurance and resistance work, IET elicited the greatest reductions in resting SBP (Cornelissen and Smart 2013, Carlson et al. 2014). The effect size is similar to that of monotherapy with betablockade (Wong and Wright 2014).

The isometric training stimulus, responsible for the reductions in resting blood pressure, probably relates to a combination of intensity ( $\%$ MVC), muscle mass (IHG vs. double-leg) and program length (number of weeks). Moreover similar blood pressure responses to isometric arm and leg exercise are observed (Williams 1991), however it is intuitive to assume that the smaller active muscle mass in arm compared to leg isometric 
exercise may induce a greater blood pressure response. Whilst the effects of different IET intensities have been investigated across a number of hemodynamic measures such as systolic, diastolic and mean-arterial blood pressures, heart rate and total peripheral resistance, there are currently only three randomized controlled trials (RCT) that have directly compared the effects of different IET intensities on resting BP (Wiles et al. 2010, Baross et al. 2012). Baross et al. (2012) and Gill et al. (2015) compared the effects of isometric bilateral leg extensions (four sets of 2 min contractions) at a lower maximal voluntary contraction (MVC) $(\sim 8 \%)$ and a higher $(\sim 14 \%$ MVC) intensity. Reductions in resting BP and concomitant vascular adaptations were observed in the high intensity group; whereas, no BP reductions or vascular adaptations were evident in the lower group suggesting the threshold for anti-hypertensive effect lies between 8-14\%, but Wiles et al. (2010) also compared the effects of isometric bilateral leg extensions on BP (four sets of 2 min contractions) at a lower ( $10 \% \mathrm{MVC})$ and a higher ( $20 \% \mathrm{MVC})$ intensity. At 4 weeks into the study no significant differences in resting BP were observed in either group, however, at the studies end ( 8 weeks) significant reductions in resting blood pressure were reported in each group. More recently, Gill et al. (2015) compared the effects of low $(\sim 23 \%$ MVC) and moderate $(\sim 34 \%$ MVC) IET over a 3 week period and reported a reduction in SBP in the moderate intensity group only. In our recent meta-analysis of IET to manage BP (Carlson et al. 2014) two studies utilized an intensity of $10 \% \mathrm{MVC}$ as a lower intensity comparison. One of these studies (Wiles et al. 2010) used leg exercise and another (Wiley et al. 1992) used handgrip exercise. The data from Wiles et al. leaves open the possibility that there may be some anti-hypertensive effect from $10 \%$ MVC (Wiles et al. 2010). Those who have participated in IET studies understand the physical and mental demands of sustaining four sets of isometric exercise at $30 \% \mathrm{MVC}$ for $2 \mathrm{~min}$. Moreover the frail or elderly, who may initially struggle with IET at $30 \% \mathrm{MVC}$, are more likely to be hypertensive and therefore derive most benefit from IET. The primary reason we are conducting this study is to establish if there exists an anti-hypertensive effect when conducting MVC at intensities of $10 \%$ or as low as $5 \%$. Our hypothesis is that we can show an anti-hypertensive effect with IET at $10 \% \mathrm{MVC}$, but not $5 \% \mathrm{MVC}$, in normotensive people, as we particularly wished to avoid conducting this initial study in populations exposed to potent vasoactive agents. If we can show an anti-hypertensive effect in normotensive participants, then intuitively these effects are also likely in older hypertensive patients. In addition, in the interests of scientific validity it would be valuable to truly establish if $10 \% \mathrm{MVC}$ or even $5 \% \mathrm{MVC}$ elicit placebo or anti-hypertensive effects. Our secondary aim was to examine if sampling blood pressure for longer periods eliminated the 'white coat hypertension' effect.

The purpose of the present study is to compare the effect of two isometric handgrip training programs performed at different intensities.

\section{Materials and Methods}

\section{Participants}

Twenty two participants, 13 males and 9 females, aged $38.8 \pm 11$ years, with resting BP within the normal range $(>90 />60 \mathrm{~mm} \mathrm{Hg}$ to $<139 /<90 \mathrm{~mm} \mathrm{Hg})$ volunteered to participate in the study. All participants were staff or students of the University of New England. Two participants (one male and one female) were unable to complete the study; both due to family circumstances. Participants were assessed as eligible if they were normotensive, had no significant visual or motor impairments, could follow verbal instructions and were between the ages of 25 and 65 years. At a screening session prior to commencement of the isometric exercise program all potential volunteers were asked to complete a participant history and medical questionnaire, an adult exercise screening questionnaire, and a written consent. At this time it was determined that participants were able to exercise and were not taking any medication that could affect the hemodynamic variables being investigated or their ability to perform IHG exercise. Baseline BP measurements were taken on 3 consecutive days, at the same time each day, prior to the commencement of training. Baseline participant characteristics are displayed in Table 1.

To minimize the influence of external variables on BP measurements, participants were asked to refrain from vigorous exercise and alcohol for $24 \mathrm{~h}$ prior to each scheduled continuous blood pressure measurement, to abstain from caffeine for $12 \mathrm{~h}$ prior and fast for $4 \mathrm{~h}$ prior. Prior to the commencement of BP measurements and IHG exercise participants were familiarized with the all equipment and IHG and BP measurement procedures. All study protocols were approved by the research ethics committee of the University of New England, HREC Approval Number HE14-047. 


\section{Study design}

Based upon the mean difference for change in SBP in our meta-analyses (Carlson et al. 2014) we calculated that 10 participants in each group were required to detect a $5 \%$ significant change with $80 \%$ power. Participants were randomly allocated to either IHG training at $10 \%$ MVC $(\mathrm{n}=10)$ or IHG training at $5 \%$ MVC $(n=10)$. Blind randomisation of participants was done using Microsoft Excel 2007 random number generator. Following group allocation all participants were instructed on the correct usage of the IHG equipment. Subsequent to group assignment all participants underwent baseline cardiovascular measures (systolic, diastolic and mean arterial blood pressure, heart rate) which were repeated each week for the duration of the training program.

\section{Arterial BP and heart rate}

Arterial BP and heart rate were continuously monitored using the Finometer (FMS, Amsterdam, Netherlands) Model-2 blood pressure monitor. The Finometer is considered to provide blood pressure information which is robust unless potent vasoactive agents are administered (McAuley et al. 1997). A wrap around finger cuff equip with, air bladder, light source (LED) and light detector (photo diode) was placed around the middle finger of the dominant hand. Care was taken when selecting and fitting an appropriate size cuff for each participant. The cuffed digit was maintained near heart level and the hydrostatic height correction unit of the Finometer compensated for any further hydrostatic pressure differences. Prior to recording cardiovascular measures the Finometer measurement of the finger arterial pressure was calibrated until the physical repetition rate recorded 40 beats or more. The Finometer provided a beat to beat estimate of the arterial waveform.

\section{IHG training protocol}

Participants in both groups completed four sets of 2 min IHG contractions 3 days per week for 6 weeks with the aid of a programmed dynamometer (DHD-3 Digital Hand Dynamometer, Saehan Corp, South Korea). Isometric contractions were performed using the nondominant hand at either $10 \%$ or $5 \%$ MVC separated by a 1 min rest period. A direct-reading light box was attached directly to the dynamometer to provide visual feedback to assist participants in maintaining the desired contraction force. The light box was calibrated to either $5 \%$ or $10 \%$ MVC for every participant every session, furthermore, the dynomometer display was monitored by an investigator each time. In terms of force exerted, our participants were able to exert a maximal handgrip force of between $30-50 \mathrm{Nm}$, so at $10 \% \mathrm{MVC}$ this equated to $3.0-5.0 \mathrm{Nm}$ and at $5 \% \mathrm{MVC}$ this was $1.5-2.5 \mathrm{Nm}$.

To avoid Valsalva manoeuvres participants were instructed to breathe at a normal rhythm and depth. Participants were seated in an erect position so that the shoulder was adducted and neutrally rotated, the elbow was flexed at a $90^{\circ}$ angle and the forearm and wrist were in a neutral position. The dynamometer was arranged in the participants' hand to ensure that it fitted comfortably, where necessary, the handle of the dynamometer was adjusted to the desired fit. Participants were instructed to apply grip force gently and smoothly and were advised by the researcher to hold their contraction once they had reached the desired IHG intensity. Participants trained every other weekday (Monday, Wednesday and Friday) with rest days in between. MVC was determined at the beginning of each training session (via imbedded electronic linear load cells contained within each handgrip); participants were asked to perform three MVCs with their non- dominant hand. The three measurements were then averaged to arrive at the MVC. On the third day of training each week, and prior to commencement of IHG training, resting BP measurements were recorded; continuous BP measurements were also recorded throughout the duration of these training sessions. Before resting $\mathrm{BP}$ measures were taken participants were asked to sit quietly for at least $10 \mathrm{~min}$. All resting BP measurements were conducted within $2 \mathrm{~h}$ of the initial baseline testing time of day. All sessions were supervised in the exercise physiology laboratory at the University of New England, Armidale, Australia.

\section{Data handling and statistical analysis}

The Finometer device computed all hemodynamic variables online and stored the data in result files on a hard disk. Calibration of all pressure transducers was maintained throughout the study. Waveform filtering and level correction protocols corrected arterial hemodynamic measures. BeatScope 1.1a software (FMS, Amsterdam, Netherlands) was used to integrate participants' gender, age, body mass and weight; this information was further integrated to compute beat-to-beat SBP and DBP values. Finometer non-invasive arterial pressure was averaged over 120,60, 30 , and $15 \mathrm{~s}$ to account for the effects of BP variability due to different sampling durations. All processed data were transferred to Microsoft Excel (Microsoft 
Corporation, Redmond, WA, USA) spread sheets.

Prior to statistical analysis all data were assessed for compliance with parametric test assumptions, where assumptions were violated transformations were applied (Tabachnick and Fidell 2013). Specifically a lower than expected outlying case was detected for a participant's post systolic blood pressure from the $10 \%$ group. To reduce the impact of the univariate outlier the deviant result was reduced to reflect a measurement one unit larger than the next most extreme score. Statistical analysis was performed using Microsoft Excel and SPSS Statistics (IBM Corporation, Armonk, NY, USA) software, version 22. We used independent sample t-tests to compare baseline BP and post intervention BP between groups and paired sample t-tests were performed to determine within groups variations from baseline to post intervention blood pressure, in addition Cohen's $d$ statistics were calculated to classify effect size. Two-way ANOVA with co-variates was performed to determine between group differences over time.

The magnitude of change in BP has been associated with initial resting values (Millar et al. 2007) to assess whether changes in resting BP following IHG training were influenced by the initial baseline BP values. Similarly in a separate analysis, age was added as a co-variate. An alpha level of $<0.05$ was determined as representing statistical significance, and the Bonferroni post-hoc procedure was used to further explore any significant differences that were detected.

\section{Results}

The adherence to IHG was $100 \%$ in the 20 participants who completed the 6 week study. Groups were matched at baseline for age (range 27-61 years), gender, body mass, SBP (range 105-134 mm Hg), DBP (range 64-84 $\mathrm{mm} \mathrm{Hg}$ ) and BMI (range 23-46) (Table 1).

As we collected $120 \mathrm{~s}$ of resting BP data we chose this as the default for our primary aim which was to see if BP was reduced. In Table 2 we have also reported 60,30 and $15 \mathrm{~s}$ rolling sample data in order to address our secondary aim of examining effect of sample duration on BP change.

\section{Systolic blood pressure}

Reductions in baseline versus post-intervention SBP were not significantly different in the $5 \% \mathrm{MVC}$ group, $-4.04 \mathrm{~mm} \mathrm{Hg}$ (95\% CI -8.67 to $+0.59, p=0.08)$, Cohen's $d$ for this test was 0.56 which can be described as a medium to large effect size. There was no significant baseline versus post-intervention reductions in the $10 \%$ MVC group, $-5.62 \mathrm{~mm} \mathrm{Hg}(95 \% \mathrm{CI}-11.5$ to $+0.29, p=0.06)$, Cohen's $d$ for this test was 0.78 which can be described as large. Post-intervention SBP was similar between the $5 \% \mathrm{MVC}$ and $10 \% \mathrm{MVC}$ groups $\quad 116.3 \pm 6 \quad \mathrm{~mm} \mathrm{Hg} \quad$ vs. $\quad 114.8 \pm 2.6 \mathrm{~mm} \mathrm{Hg}$ respectively $F(1,17)=0.45, \quad p=0.51$. No interaction effects were observed.

\section{Diastolic blood pressure}

Changes in baseline versus post-intervention DBP were not significantly different in the $5 \% \mathrm{MVC}$ group, $-0.97 \mathrm{~mm} \mathrm{Hg}(95 \% \mathrm{CI}-2.56$ to $+0.62, p=0.20)$, $d=0.18$. Similarly, there were no significant baseline versus post-intervention difference in the $10 \% \mathrm{MVC}$ group, $+1.8 \mathrm{~mm} \mathrm{Hg}(95 \% \mathrm{CI}-1.29$ to $+4.89, p=0.22)$, $d=0.26$ which can be described as small. Postintervention DBP was similar between the $5 \% \mathrm{MVC}$ and $10 \% \mathrm{MVC}$ groups $68.5 \pm 4 \mathrm{~mm} \mathrm{Hg}$ vs. $69.4 \pm 7 \mathrm{~mm} \mathrm{Hg}$ respectively $F(1,17)=0.72, p=0.41$. No interaction effects were observed.

\section{Heart rate}

There were no significant changes in heart rate during the 6 week study. Heart rate did not increase during IHG exercise $>10$ beats. $\mathrm{min}^{-1}$ in any participant during any session.

\section{Effect of sampling duration}

ANOVA showed that using $120 \mathrm{~s}$ produced significantly higher SBP and DBP than using 15, 30 or 60 s sampling durations (Table 2).

Table 1. Resting baseline data.

\begin{tabular}{lcc}
\hline & $\mathbf{5 \%}(\mathbf{n = 1 0})$ & $\mathbf{1 0} \% \mathbf{( n = 1 0 )}$ \\
\hline Age (years) & $38.8 \pm 10.5$ & $38.7 \pm 12.6$ \\
Male $(\mathrm{n})$ & 6 & 6 \\
Female $(\mathrm{n})$ & 4 & 4 \\
Height $(\mathrm{m})$ & $1.72 \pm 0.1$ & $1.75 \pm 0.1$ \\
Weight $(\mathrm{kg})$ & $83.3 \pm 21.4$ & $85.3 \pm 26.9$ \\
BMI & $28.4 \pm 7.7$ & $27.7 \pm 7.4$ \\
RSBP $(\mathrm{mm} \mathrm{Hg})$ & $120.3 \pm 8.1$ & $120.5 \pm 11.8$ \\
RDBP $(\mathrm{mm} \mathrm{Hg})$ & $69.5 \pm 6.4$ & $67.6 \pm 6.4$ \\
\hline
\end{tabular}

All values are reported as means. BMI - body mass index, RSBP - resting systolic blood pressure, RDBP - resting diastolic blood pressure. All $p>0.05$. 
Table 2. Mean difference scores for continuous blood pressure measurement.

\begin{tabular}{lcccc}
\hline & & \multicolumn{2}{c}{ Blood pressure at 120 s } \\
& Pre SBP & Post SBP & Pre DBP & Post DBP \\
\hline Blood pressure & & & & $-0.09 \pm 1.25$ \\
$60 s$ & $0.57 \pm 0.75$ & $0.02 \pm 1.81$ & $0.39 \pm 0.54$ & $0.03 \pm 2.88$ \\
$30 s$ & $0.91 \pm 1.76$ & $-0.09 \pm 3.46$ & $0.48 \pm 0.95$ & $0.17 \pm 4.21$ \\
\hline
\end{tabular}

t statistic: $* p<0.05, * * p<0.01$. Data are means $\pm \mathrm{SD}$.

Age as a covariate

An ANOVA indicated that $20 \%$ of the variance in post training SBP was explained by the participant ages, $F(1,17)=4.30, p=0.05$, partial $\eta^{2}=0.202$. Furthermore, $20 \%$ of the variance in post training DBP was also explained by participant ages, $F(1,17)=4.31$, $p=0.05$, partial $\eta^{2}=0.202$.

\section{Discussion}

Our work examines the effects of the lowest isometric training intensities that have been explored. Our primary finding was that $\mathrm{IHG}$ training performed at either $5 \% \mathrm{MVC}$ or $10 \% \mathrm{MVC}$ elicited no statistically significant reduction on either SBP or DBP after 6 weeks of training in normotensive adult men and women. However, our results did show a reduction in SBP in both the $5 \%$ and $10 \%$ groups and this was supported by medium to large effect sizes suggesting that IHG training at both intensities has an anti-hypertensive effect. In the current study, after 6 weeks of IHG training, reductions in SBP were similar to the antihypertensive effects observed in monotherapy of 5-7 mm Hg (Wong and Wright 2014). Reductions in SBP and DBP of $\geq 2 \mathrm{~mm} \mathrm{Hg}$ can significantly reduce the incidence of CVD in both hypertensive and normotensive individuals, subsequently small reductions of this magnitude are considered clinically meaningful (Collaboration 2003, Wong and Wright 2014). We identified one other published RCT that did not detect statistically significant reductions in resting BP yet were able to report clinically meaningful reductions in ambulatory SBP and night-time SBP ( 3-4 mm Hg) (Stiller-Moldovan et al. 2012). Our secondary finding was that there was minimal effect of different sampling durations for rolling averages (assessed over 120, 60, 30 and $15 \mathrm{~s}$ ) on pre- or post-blood pressure values.

Our primary finding was no statistically significant effect on either SBP or DBP after 6 weeks of training in normotensive adults. If one assumes for arguments' sake that there exists a $2 / 3$ relative intensity of isometric handgrip (IHG) exercise to Double Leg (DL) isometric exercise, then the comparison of our data (nonsignificant changes after 6 weeks of IHG at $10 \%$ MVC) might actually be showing similar results as Wiles et al. (2010) who used $10 \% \mathrm{MVC}$ DL for 8 weeks. Thus, Wiles et al. (2010) used a slightly higher intensity (comparing 'like-for-like' IHG to DL, but in a longer program). This interpretation is further confirmed by the 4-weeks to 6-weeks comparison between the data of Wiles et al. (2010) and the current data (both being nonsignificant). However, it should be noted that Wiles et al. (2010) did report significant reductions in resting BP at $\sim 10 \%$ MVC after 8 weeks, but not after 4 weeks of training.

Contrary to the findings of Wiles et al. (2010), Howden et al. (2002) observed statistically significant reductions in SBP after 3 weeks of isometric leg exercise at $\sim 20 \% \mathrm{MVC}$ and after 4 weeks of isometric arm training at $\sim 30 \%$ MVC. Furthermore, Gill et al. (2015) hypothesized that reductions in resting BP from IET would be intensity dependent, they compared the effects of low ( $23 \%$ MVC) and moderate ( $34 \%$ MVC) IET over a 3 week period and reported a reduction in SBP in the moderate intensity group only. Baross et al. (2012) found that $\sim 14 \%$ MVC, but not $\sim 8 \%$ MVC elicited BP reductions after 8 weeks of isometric bilateral leg extension exercise. Collectively, these studies highlight a relationship between IET intensity, time (study duration) and reductions in resting BP. They suggest that the magnitude and rate at which resting BP is reduced increases as exercise intensity increases and that where present these reductions will continue to increase over time, with 8-10 weeks being the longest durations investigated so far. Subsequently, in terms of the current study, it could be the case that at intensity levels as low as 
$5 \%$ and $10 \%$ MVC that 6 weeks of IHG training is not a sufficiently long enough training period to elicit statistically significant results, but may elicit clinically significant results. Whilst the relationship between IET intensity, time and BP reductions are not fully understood, together, our findings and those of others suggest an anti-hypertensive threshold that may be well below $10 \%$ MVC.

The precise mechanism(s) of the antihypertensive effect(s) of isometric exercise remain unclear; however, it appears likely that the true stimulus is probably a result of the competing drives of reduced blood flow (from external compression of the blood vessels and sympathetic vasoconstriction) in the face of a vasodilator stimulus from metabolite accumulation. The variation in individual adaptation rates to IET are probably due to the individual variations in plasma noradrenaline levels, which exist prior to training (Esler 2011). Indeed, plasma noradrenaline levels have been shown to be linked to training-induced reductions in resting blood pressure (especially in hypertensives, who seemingly have higher levels of plasma noradrenaline) (Cornelissen and Fagard 2005).

Previous work has suggested that blood flow is fully occluded at approximately 55-75\% of MVC, but those able to exert more handgrip force will have higher occlusion thresholds (Barnes 1980). Intuitively even at lower percentage of MVC with arm or leg isometric exercise, partial occlusion of blood flow is likely. The reactive hyperemia may therefore be due to partial or full occlusion of the brachial artery during 2 min IHG efforts. Previous work has postulated that in response to ischemia induced by IHG activity a number of metabolites are produced; these include prostaglandins, beta-endorphins and HIF-alpha (Wong et al. 2015, Stiller-Moldovan et al. 2012, Wong and Wright 2014). It may be that the presence or absence of reactive hyperemia is determined by the MVC intensity during isometric exercise. Moreover, the results of our work and of others such as Wiles et al. (2010) and Baross et al. (2012) suggest that MVC intensities as little as 5-14\% may induce sufficient, albeit partial, occlusion to blood flow to induce ischemia and the subsequent metabolite production that may be contributing to BP reductions. Nonetheless, IET response rates are highly variable between individual participants whereby some respond to IET (in highly varying degrees) and others do not (McGowan et al. 2007, StillerMoldovan et al. 2012). Whilst the reasons for this are not fully understood there are a number of variables that are likely to affect the magnitude of reduction in resting BP. For instance, the greatest isometric exercise reductions in resting $\mathrm{BP}$ have been observed in pre-hypertensive and hypertensive individuals (Wiley et al. 1992, Taylor et al. 2003) and magnitude of change in BP has been associated with initial resting $\mathrm{BP}$ values such that greater reductions are observed in individuals with higher pre-IET BP (Millar et al. 2007). In the current study participant age was also identified as a significant predictor of resting BP reduction in response to IET, this finding was not surprising as age is related to arterial stiffness and hypertension.

Our secondary aim was to assess whether different sampling durations for rolling averages affected BP values for continuous BP measurements. Due to the transient nature of blood pressure the recommended gold standard procedure for measuring blood pressure is via $24 \mathrm{~h}$ ambulatory monitoring, however, ambulatory means of BP measurement were unavailable to this study. In an attempt to attenuate the natural fluctuations of BP we used a continuous BP measurement and also measured baseline resting BP on three separate occasions for a 2 min period each time. We found that in all cases $120 \mathrm{~s}$ sampling data was significantly higher than for the other sampling durations. We believe that the longer $120 \mathrm{~s}$ sampling duration provides a more robust default for our primary analysis.

The lack of a non-exercising or 'sham IRT' control group was a limitation in this study.

\section{Conclusion}

This is the first threshold (MVC \%) examination for antihypertensive responses to IET at intensities traditionally used as a control or placebo. In those people unable to complete IET at the traditional intensity of $30 \% \mathrm{MVC}$, our results suggest that IET intensities of both $5 \%$ and $10 \%$ MVC may offer clinically relevant anti-hypertensive effects, despite the absence of statistically significant reductions in resting BP. As this is the first study of its kind, the study duration and the sample size may have been insufficient to demonstrate a statistically significant reduction in resting BP at these low intensities. Further research involving a larger participant cohort, conducted over a longer period of time is required to determine a minimum therapeutic threshold for antihypertensive response, this determination would aid in the design of future RCT's to determine if sham groups are truly that. Moreover, ascertaining the 
minimum IET intensity for $\mathrm{BP}$ reduction may also be important in terms of designing exercise programs for the frail and elderly. Elderly people may struggle to complete IHG exercise at $30 \% \mathrm{MVC}$, and why would we ask them to in light of our clinically significant findings.

\section{Conflict of Interest}

There is no conflict of interest.

\section{Acknowledgements}

The authors thank Dr Gudrun Dieberg for her assistance with study design.

\section{References}

BADROV MB, BARTOL CL, DiBARTOLOMEO MA, MILLAR PJ, MCNEVIN NH, MCGOWAN CL: Effects of isometric handgrip training dose on resting blood pressure and resistance vessel endothelial function in normotensive women. Eur J Appl Physiol 113: 2091-2100, 2013 a.

BADROV MB, HORTON S, MILLAR PJ, MCGOWAN CL: Cardiovascular stress reactivity tasks successfully predict the hypotensive response of isometric handgrip training in hypertensives. Psychophysiology 50: 407-414, 2013b.

BARNES WS: The relationship between maximum isometric strength and intramuscular circulatory occlusion. Ergonomics 23: 351-357, 1980.

BAROSS AW, WILES JD, SWAINE IL: Effects of the intensity of leg isometric training on the vasculature of trained and untrained limbs and resting blood pressure in middle-aged men. Int J Vasc Med 2012: 964697, 2012.

CARLSON DJ, DIEBERG G, HESS NC, MILLAR PJ, SMART NA: Isometric exercise training for blood pressure management: a systematic review and meta-analysis. Mayo Clin Proc 89: 327-334, 2014.

COLLABORATION BPLTT: Effects of different blood-pressure-lowering regimens on major cardiovascular events: results of prospectively-designed overviews of randomised trials. Lancet 362: 1527-1535, 2003.

CORNELISSEN VA, FAGARD RH: Effects of endurance training on blood pressure, blood pressure-regulating mechanisms, and cardiovascular risk factors. Hypertension 46: 667-675, 2005.

CORNELISSEN VA, SMART NA: Exercise training for blood pressure: a systematic review and meta-analysis. $J \mathrm{Am}$ Heart Assoc 2: e004473, 2013.

DEVEREUX GR, WILES JD, SWAINE I: Markers of isometric training intensity and reductions in resting blood pressure. J Sports Sci 29: 715-724, 2011.

ESLER M: The sympathetic nervous system through the ages: from Thomas Willis to resistant hypertension. Exp Physiol 96: 611-622, 2011.

GILL KF, ARTHUR ST, SWAINE I, DEVEREUX GR, HUET YM, WIKSTROM E, CORDOVA ML, HOWDEN R: Intensity-dependent reductions in resting blood pressure following short-term isometric exercise training. J Sports Sci 33: 616-621, 2015.

HAJJAR I, KOTCHEN TA: Trends in prevalence, awareness, treatment, and control of hypertension in the United States, 1988-2000. JAMA 290: 199-206, 2003.

HOWDEN R, LIGHTFOOT JT, BROWN SJ, SWAINE IL: The effects of isometric exercise training on resting blood pressure and orthostatic tolerance in humans. Exp Physiol 87: 507-515, 2002.

JAMES PA, OPARIL S, CARTER BL, CUSHMAN WC, DENNISON-HIMMELFARB C, HANDLER J, LACKLAND DT, LEFEVRE ML, MACKENZIE TD, OGEDEGBE O, SMITH SC JR, SVETKEY LP, TALER SJ, TOWNSEND RR, WRIGHT JT JR, NARVA AS, ORTIZ E: 2014 evidence-based guideline for the management of high blood pressure in adults: report from the panel members appointed to the Eighth Joint National Committee (JNC 8). JAMA 311: 507-520, 2014.

MCAULEY D, SILKE B, FARRELL S: Reliability of blood pressure determination with the Finapres with altered physiological states or pharmacodynamic conditions. Clin Auton Res 7: 179-184, 1997.

MCGOWAN C, LEVY A, MCCARTNEY N, MACDONALD M: Isometric handgrip training does not improve flowmediated dilation in subjects with normal blood pressure. Clin Sci 112: 403-409, 2007. 
MILLAR PJ, BRAY SR, MCGOWAN CL, MACDONALD MJ, MCCARTNEY N: Effects of isometric handgrip training among people medicated for hypertension: a multilevel analysis. Blood Press Monit 12: 307-314, 2007.

MILLAR PJ, BRAY SR, MACDONALD MJ, MCCARTNEY N: The hypotensive effects of isometric handgrip training using an inexpensive spring handgrip training device. J Cardiopulm Rehabil Prev 28: 203-207, 2008.

STILLER-MOLDOVAN C, KENNO K, MCGOWAN CL: Effects of isometric handgrip training on blood pressure (resting and $24 \mathrm{~h}$ ambulatory) and heart rate variability in medicated hypertensive patients. Blood Press Monit 17: 55-61, 2012.

TABACHNICK BG, FIDELL LS: Using Multivariate Statistics. Pearson \& Allyn and Bacon, Boston, 6th Edn., 2013.

TAYLOR AC, MCCARTNEY N, KAMATH MV, WILEY RL: Isometric training lowers resting blood pressure and modulates autonomic control. Med Sci Sports Exerc 35: 251-256, 2003.

WILES JD, COLEMAN DA, SWAINE IL: The effects of performing isometric training at two exercise intensities in healthy young males. Eur J Appl Physiol 108: 419-428, 2010.

WILEY RL, DUNN CL, COX RH, HUEPPCHEN NA, SCOTT MS: Isometric exercise training lowers resting blood pressure. Med Sci Sports Exerc 24: 749-754, 1992.

WILLIAMS CA: Effect of muscle mass on the pressor response in man during isometric contractions. $J$ Physiol 435 : 573-584, 1991.

WONG GW, WRIGHT JM: Blood pressure lowering efficacy of nonselective beta-blockers for primary hypertension. Cochrane Database Syst Rev 2: CD007452, 2014.

WONG GW, LAUGEROTTE A, WRIGHT JM: Blood pressure lowering efficacy of dual alpha and beta blockers for primary hypertension. Cochrane Database Syst Rev 8: CD007449, 2015. 